Joyce Mendes de Andrade Schramm 1 Célia Landmann Szwarcwald 2

\section{Diferenciais nas taxas de mortalidade neonatal e natimortalidade hospitalares no Brasil: um estudo com base no Sistema de Informações Hospitalares do Sistema Único de Saúde (SIH/SUS)}

\author{
Differences between neonatal mortality \\ and stillbirth rates in Brazil: a study based \\ on the Unified Health System (SIH/SUS) \\ Hospital Information System
}

1 Departamento de Epidemiologia e Métodos Quantitativos em Saúde, Escola Nacional de Saúde Pública, Fundação Oswaldo Cruz Rua Leopoldo Bulhões 1480, 8 o andar, Rio de Janeiro, $R J$ 21041-210, Brasil. joyce@ensp.fiocruz.br 2 Departamento de Informações em Saúde, Centro de Informação Científica e Tecnológica, Fundação Oswaldo Cruz Av. Brasil 4365,

Rio de Janeiro, $R J$ 21045-900, Brasil. celia@malaria.procc.fiocruz.br

\begin{abstract}
The main objective of this article is to estimate stillbirth and neonatal mortality rates in Brazilian States based upon the country's Hospital Information System. Analysis of 1995 data reveals contrasting rates between the various regions of the country. In order to elucidate the States' different rates, we focused on the association between indicators of coverage, utilization, and access to the Unified Health System (SUS). The results for the neonatal period mostly showed higher early neonatal mortality rates when compared to late neonatal mortality rates, higher neonatal mortality rates in the States comprising the South and Southeast regions, less variable rates between those States, and extremely low rates in some States of the North, Central-West, and Northeast regions. The limited supply of SUS services and low access to same are relevant constraints on health care for the population in the North and Northeast. Aspects related to quality of childbirth and neonatal care are also reflected in the rates studied. The findings suggest that spatial and temporal monitoring of these rates could provide analytical support for organizing the Maternal and Child Health Program.
\end{abstract}

Key words Fetal Death; Neonatal Mortality; Hospital Information Systems

Resumo Este trabalho tem como objetivo estimar as taxas de natimortalidade e mortalidade neonatal a partir do SIH/SUS nas Unidades da Federação para o ano de 1995. Observaram-se os contrastes regionais entre as taxas estudadas. As diferenças entre os Estados foram apreciadas quanto à associação com indicadores de cobertura, de utilização e de acesso aos serviços do SUS. Os resultados correspondentes às taxas do período neonatal revelam: predominância de valores mais elevados para o componente neonatal precoce; maior homogeneidade e maiores valores dos coeficientes de mortalidade neonatal para as Unidades Federadas que integram as regiões Sul e Sudeste; valores extremamente baixos para alguns Estados das regiões Norte, Centro-Oeste e Nordeste. Observou-se que a baixa oferta e o acesso limitado aos serviços do SUS são restrições relevantes para a população residente nas regiões Norte e Nordeste. Aspectos relacionados à qualidade da assistência ao parto e ao recém-nascido, igualmente, estão refletidos nas taxas em estudo. Os achados sugerem que o monitoramento espaço-temporal das taxas hospitalares pode fornecer subsídios importantes para a organização do programa materno-infantil. Palavras-chave Morte Fetal; Mortalidade Neonatal; Sistemas de Informação Hospitalar 


\section{Introdução}

No Brasil, a mortalidade infantil tem apresentado acentuado declínio a partir da segunda metade da década de 80 , atribuído, principalmente, à diminuição dos óbitos no período pós-neonatal (28 dias e mais) que apresenta maior suscetibilidade às ações preventivas como o saneamento básico e imunizações (Becker, 1991). Já no que diz respeito à mortalidade neonatal, estudos em alguns estados brasileiros evidenciaram pequeno decréscimo, ou até mesmo estabilidade, sobretudo para o componente neonatal precoce (Souza \& Gotlieb, 1993; Bercini, 1994; Leal \& Szwarcwald, 1996).

Esta situação se diferencia daquela dos países com reconhecido padrão de redução da mortalidade infantil, onde os óbitos infantis no período neonatal têm diminuído significativamente. A organização dos cuidados pré-natais em níveis de atenção e a adequação do local de nascimento em relação aos riscos mensuráveis da gravidez, portanto do binômio mãecriança, têm sido considerados como os principais fundamentos da política de organização da atenção perinatal nesses países (Campbell \& MacFarlane, 1986; Shenai et al., 1991; Groupe Image, 1997).

A mortalidade infantil e seus componentes ganham um lugar de destaque entre os eventos sentinela, definidos por Rutstein et al. (1976) como "sinais de alerta" de possíveis falhas na qualidade da atenção. Estudos recentes no país sobre a mortalidade perinatal e neonatal constataram um excesso relevante de mortalidade considerada evitável pelo adequado controle da gravidez, adequada atenção ao parto e diagnóstico e tratamento precoce (Barros et al., 1987; Leal \& Szwarcwald, 1996). A fragilidade dos serviços de saúde em diferenciarem a gravidade das doenças, a dificuldade de acesso em obter os cuidados necessários e as responsabilidades obstétrica e pediátrica foram igualmente identificados como fatores que contribuíram para as causas de mortes infantis no período neonatal (Carvalho, 1993; Aragão, 1994; Salinas et al., 1997).

Entretanto, o sub-registro das estatísticas do Registro Civil, tanto de nascimentos como de óbitos, assim como a subenumeração das estatísticas vitais dos sistemas do Ministério da Saúde (Sistema de Informações sobre Mortalidade e Sistema de Nascimentos) dificultam as estimativas do coeficiente de mortalidade infantil e de seus componentes no país (Barros et al., 1985; Szwarcwald, 1997).

Neste contexto, reconhecendo a importância de aprofundar o estudo de um conjunto de aspectos hospitalares para melhor compreen- der e definir estratégias para reduzir o componente neonatal, Schramm \& Szwarcwald (2000) estudaram o Sistema de Informações Hospitalares do Sistema Único de Saúde (SIH/SUS) como fonte alternativa para estimar a natimortalidade e a mortalidade neonatal precoce hospitalar. Concluíram que o SIH/SUS constitui-se em uma fonte oportuna para o cálculo das taxas de natimortalidade e mortalidade neonatal hospitalares, tendo em vista não só a cobertura incompleta das estatísticas vitais no Brasil, mas também a agilidade do SIH/SUS em disponibilizar as informações em meio magnético.

Dando continuidade à proposta metodológica apresentada anteriormente por Schramm \& Szwarcwald (2000), neste trabalho, são calculados os componentes da mortalidade neonatal e os coeficientes de natimortalidade para todas as Unidades Federadas e regiões do país, utilizando o Sistema de Informações Hospitalares (SIH/SUS) como fonte de dados. Busca-se explicar as diferenças encontradas nos coeficientes por Unidade Federada por meio de indicadores que expressem a cobertura e a utilização da rede hospitalar do Sistema Único de Saúde. Os resultados são também comparados aos coeficientes de mortalidade neonatal obtidos para um conjunto de países especificamente selecionado para este fim.

\section{Metodologia}

O formulário AIH (Autorização de Internação Hospitalar) é o documento central do sistema e caracteriza a internação no Sistema Único de Saúde, nos casos de transferências entre clínicas de especialidades diferentes na mesma unidade hospitalar, ou nos casos de transferências para outras unidades hospitalares. A AIH tem a função de autorizar a realização da internação propriamente dita, para a efetivação de um procedimento clínico ou cirúrgico pré-definido; de identificar o paciente, registrar o conjunto de procedimentos médicos, serviços de diagnose e terapia realizados e servir como documento de faturamento pela unidade hospitalar ao órgão financiador dos serviços (Melo, 1996).

Atualmente, a AIH é preenchida em meio magnético (MS, 1995). Os dados são processados pelo Departamento de Informática do Sistema Único de Saúde (DATASUS), compactados e disponibilizados em CD-ROM, denominados, respectivamente, de AIH-reduzida e $\mathrm{AIH}$ mês a mês, de acordo com o tipo de arquivo e o número de variáveis incluídas.

A AIH1 é utilizada como matriz das demais AIHs. É preenchida quando ocorre uma inter- 
nação na rede hospitalar vinculada ao SUS e tem validade por um período de até 45 dias, o que a caracteriza como autorização de internação para pacientes agudos. A AIH5 é usada para dar continuidade às informações de pacientes psiquiátricos e crônicos.

Alguns aspectos metodológicos referentes à tabulação do número de nascidos vivos, nascidos mortos e óbitos registrados nas AIHs das mães são de interesse específico para este trabalho. Estes campos devem ser preenchidos nas internações por parto. Os natimortos são registrados diretamente no campo correspondente ao número de nascidos mortos. No caso dos recém-natos que morrem por alguma causa que não tenha originado uma internação prévia (condição que origina o preenchimento de uma $\mathrm{AIH}$ ), o óbito é registrado na $\mathrm{AIH}$ da mãe, no campo que especifica a condição de saída da criança. Segundo as normas do SUS, não são preenchidas AIHs para as crianças nascidas de mães diabéticas que não apresentam causa justificada para internação; com graus leves de icterícia ou potencialmente infectadas (A. W. G. Lima, comunicação pessoal).

Considerando-se as especificidades apontadas acima, o banco de dados foi preparado tomando-se por base a AIH mês a mês para o ano de 1995 e para todos os estados do país. Todas as internações ocorridas e classificadas no capítulo correspondente à "Gravidez, Parto e Puerpério”, CID 9a Revisão (OMS, 1985), com o preenchimento de pelo menos uma das condições no campo de "parto", "nascido morto" ou "nascido vivo", foram selecionadas assim como todas as internações ocorridas nos menores de um ano de idade. Foram processadas cerca de três milhões de autorizações hospitalares para todo o país.

Todos os casos registrados no campo "saída-óbito" (registros dos óbitos na AIH da mãe de crianças nascidas vivas que foram a óbito sem gerar uma AIH de internação própria), que tinham sido registrados ao mesmo tempo como natimortos, isto é, casos considerados como de duplo registro, foram excluídos da análise.

Para o cálculo da idade de crianças que foram internadas e morreram no período neonatal na rede hospitalar do SUS, utilizou-se um algoritmo baseado na diferença entre as datas de saída (no caso data de ocorrência do óbito) e a data de nascimento da criança.

Pressupondo-se que os óbitos no período neonatal captados pelo Sistema Único de Saúde correspondem aos filhos de gestantes que se internaram em um hospital do SUS para o parto, foi possível estimar o número de nascidos vivos, selecionando-se todas as AIHs preenchidas para os procedimentos "parto" cujo campo correspondente ao número de nascidos vivos estivesse preenchido.

$\mathrm{Na}$ impossibilidade de classificar as perdas fetais em precoces e tardias pela inexistência no banco de dados de um critério que permitisse distingui-las, o cálculo da taxa de natimortalidade incluiu no numerador todos os registros correspondente ao campo "número de nascidos mortos" da AIH. Já no denominador foram computados todos os nascimentos.

A taxa de mortalidade neonatal tardia foi estimada pela razão entre o número de óbitos ocorridos no período de 7 a 27 dias (cuja idade foi corrigida pelo algoritmo desenvolvido) e o número de nascidos vivos registrados no SIH/ SUS. Para o cálculo da mortalidade neonatal precoce, estimada pela razão entre o número de óbitos ocorridos de 0 a 6 dias e o número de nascidos vivos registrados no SIH/SUS, no numerador foram incluídos todos os óbitos registrados nas AIHs das crianças no período de 0 a 6 dias (após a correção da idade) que foram somados aos óbitos ocorridos após o nascimento e registrados nas AIHs das mães.

Para buscar possíveis explicações para a variação das taxas de mortalidade neonatal e natimortalidade entre as Unidades da Federação (UF), construiu-se um conjunto de indicadores: - Cobertura dos nascimentos pelo Sistema Único de Saúde - A cobertura foi estimada pela proporção de nascimentos ocorridos no SUS em relação ao número de nascidos vivos estimado a partir dos dados do Censo de 1991 (IBGE, 1991), usando o método de Brass (UN, 1983);

- Proporção de altas obstétricas (parto normal e cesariana) com 1 dia de internação (tempo de permanência igual a 1) - Este indicador foi obtido pela razão entre o número de altas com até um dia de internação e o número total de altas ocorridas, multiplicado por 100;

- Proporção de óbitos neonatais precoces registrados nas AIHs das mães - Calculado pela razão entre o número de óbitos registrados nas AIHs das mães e o número total de óbitos neonatais precoces, multiplicado por 100;

- Taxa de utilização do SUS no período neonatal precoce - Estimada pelo quociente entre o número de crianças com menos de sete dias que se internaram em hospitais do SUS e o número de nascidos vivos estimado a partir dos dados do Censo de 1991 (IBGE, 1991), usando o método de Brass (UN, 1983);

- Proporção de partos domiciliares - Calculada pela razão entre o número de partos ocorridos em domicílio e o número total de partos no ano de 1995, multiplicado por 100 (IBGE, 1998).

A seguir, as correlações de Pearson entre os indicadores propostos e as taxas de natimorta- 
lidade e mortalidade neonatal precoce e tardia foram investigadas e testadas quanto à significância.

Considerando-se que as mortes infantis em países desenvolvidos são essencialmente hospitalares, selecionou-se para fins de comparação, um conjunto de coeficientes de mortalidade neonatal em diversos países disponibilizados na publicação da Organização Mundial de Saúde para o ano de 1995 (WHO, 1995).

Todos os dados foram processados pelos programas computacionais $S A S$ versão 6.12 (SAS Institute, 1998) e SPSS, versão 8.0 (SPSS Incorporation, 1995).

\section{Resultados}

Os resultados referentes às taxas de natimortalidade e de mortalidade neonatal, precoce e tardia, calculados com base no SIH/SUS pela metodologia proposta, estão dispostos na Tabela 1 .

Em relação às taxas de natimortalidade, a região Sudeste foi aquela que apresentou a maior taxa, seguida da região Nordeste. Chamam atenção os valores extremamente baixos encontrados para os Estados de Rondônia, Acre, Amapá, Tocantins e Piauí quando comparados aos valores calculados para as outras Unidades Federadas do país. Da mesma forma, os valores elevados obtidos nos Estados do Maranhão e Sergipe merecem destaque. Outros contrastes podem ser citados, como as estimativas das taxas de alguns estados das regiões Norte, Nordeste e Centro-Oeste comparadas àquelas dos Estados de São Paulo e Rio Grande do Sul.

Os resultados correspondentes às taxas do período neonatal revelam: predominância de valores mais elevados para o componente neonatal precoce, quando comparados às taxas do componente neonatal tardio; maior homogeneidade e maiores valores dos coeficientes de mortalidade neonatal para as Unidades Federadas que integram as regiões Sul e Sudeste; valores extremamente baixos para alguns estados das regiões Norte, Centro-Oeste e Nordeste, em particular, para o componente neonatal precoce.

Na Tabela 2, encontram-se os indicadores utilizados neste trabalho, calculados por Unidade Federada. Com os resultados, pode-se apontar que as freqüências de partos domiciliares são muito mais elevadas nos Estados que integram as regiões Norte e Nordeste. Nos Estados do Amazonas, Pará, Maranhão e Bahia, os percentuais são superiores a $14 \%$. Nas Uni- dades Federadas das regiões Norte e Nordeste são igualmente observados os maiores percentuais de altas hospitalares de gestantes com um dia de internação. Contudo, os valores muito baixos encontrados para os Estados do Maranhão e Paraíba, quando comparados aos outros Estados da Região Nordeste, merecem ênfase. Da mesma forma, as proporções estimadas para os Estados de Minas Gerais, Paraná e Mato Grosso do Sul apresentam valores muito diferenciados dos obtidos nos outros Estados das respectivas macrorregiões.

As análises dos indicadores "taxa de utilização para o período neonatal precoce" e a "cobertura dos nascimentos pelo Sistema Único de Saúde" mostraram as disparidades existentes entre as Regiões Norte e Nordeste e as outras macrorregiões. Chama atenção o valor encontrado para a taxa de utilização do Distrito Federal que é a mais alta do país (Tabela 2).

Mediante os percentuais de óbitos neonatais precoces registrados nas AIHs das mães apresentados também na Tabela 2 , pode-se verificar que a Região Sudeste é aquela que apresenta o valor mais elevado do país, chegando a ultrapassar a média nacional em cerca de 10 pontos percentuais. Destacam-se, ainda, as proporções encontradas para os Estados de Roraima e Tocantins, cuja quase totalidade dos óbitos neonatais precoces, que ocorreram nos hospitais do SUS no mesmo período, foram registrados nas AIHs das mães. Por outro lado, o valor zero estimado para o Amapá aponta exatamente o oposto, isto é, todos os óbitos neonatais precoces ocorreram em crianças que geraram uma internação no SUS.

A Tabela 3 apresenta a matriz de correlações entre os indicadores propostos e as taxas de mortalidade, na qual podemos destacar os principais resultados que apresentaram existência de associação estatística no nível de significância de 5\%: correlação direta entre as taxas de natimortalidade e mortalidade neonatal precoce. Esta última apresentou associação positiva com a "proporção de óbitos neonatais precoces registrados nas AIHs das mães"; correlação inversa da "cobertura dos nascimentos do Sistema Único de Saúde” com a "proporção de partos domiciliares" e com a "proporção de altas obstétricas com até um dia de internação". Já a proporção de partos domiciliares apresentou associação positiva com a "proporção de altas obstétricas com até um dia de internação" e associação negativa com a "taxa de utilização do SUS no período neonatal".

Na Tabela 4, pode-se comparar as taxas estimadas para os dois componentes da mortalidade neonatal com base no SIH/SUS, para as 
Taxas de natimortalidade, mortalidade neonatal precoce e mortalidade neonatal tardia.

\begin{tabular}{|c|c|c|c|c|}
\hline $\begin{array}{l}\text { Unidade } \\
\text { da Federação }\end{array}$ & $\begin{array}{l}\text { Natimortalidade } \\
\text { neonatal precoce }\end{array}$ & $\begin{array}{l}\text { Mortalidade } \\
\text { neonatal tardia }\end{array}$ & $\begin{array}{l}\text { Mortalidade } \\
\text { neonatal }\end{array}$ & Mortalidade \\
\hline Rondônia & 8,4 & 7,7 & 1,8 & 9,5 \\
\hline Acre & 8,4 & 2,0 & 0,4 & 2,4 \\
\hline Amazonas & 15,3 & 10,2 & 1,5 & 11,7 \\
\hline Roraima & 15,2 & 6,5 & 2,6 & 9,1 \\
\hline Pará & 12,1 & 6,1 & 1,8 & 7,8 \\
\hline Amapá & 7,1 & 1,2 & 1,3 & 2,5 \\
\hline Tocantins & 4,9 & 5,5 & 1,1 & 6,6 \\
\hline Norte & 11 & 7 & 2 & 8 \\
\hline Maranhão & 22,7 & 3,4 & 0,9 & 4,4 \\
\hline Piauí & 8,0 & 2,6 & 0,8 & 3,4 \\
\hline Ceará & 12,1 & 8,8 & 2,2 & 11,1 \\
\hline Rio Grande do Norte & 12,3 & 7,5 & 1,8 & 9,4 \\
\hline Paraíba & 12,1 & 8,8 & 2,8 & 11,5 \\
\hline Pernambuco & 14,1 & 7,6 & 2,5 & 10,1 \\
\hline Alagoas & 14,5 & 6,0 & 2,5 & 8,4 \\
\hline Sergipe & 21,5 & 11,4 & 2,7 & 14,1 \\
\hline Bahia & 12,4 & 8,4 & 1,9 & 10,4 \\
\hline Nordeste & 14 & 7 & 2 & 9 \\
\hline Minas Gerais & 17,3 & 10,4 & 2,2 & 12,6 \\
\hline Espírito Santo & 19,9 & 8,1 & 2,4 & 10,5 \\
\hline Rio de Janeiro & 17,7 & 9,1 & 2,2 & 11,3 \\
\hline São Paulo & 13,4 & 12,0 & 3,0 & 15,0 \\
\hline Sudeste & 16 & 11 & 3 & 13 \\
\hline Paraná & 12,7 & 8,2 & 2,2 & 10,4 \\
\hline Santa Catarina & 12,5 & 9,9 & 2,2 & 12,1 \\
\hline Rio Grande do Sul & 9,7 & 8,0 & 3,2 & 11,2 \\
\hline Sul & 12 & 8 & 3 & 11 \\
\hline Mato Grosso do Sul & 13,8 & 10,1 & 2,5 & 12,6 \\
\hline Mato Grosso & 15,1 & 5,7 & 1,6 & 7,3 \\
\hline Goiás & 11,8 & 8,1 & 3,1 & 11,2 \\
\hline Distrito Federal & 10,2 & 7,5 & 2,5 & 10,0 \\
\hline Centro-oeste & 13 & 8 & 2 & 10 \\
\hline Brasil & 14 & 8 & 2 & 11 \\
\hline
\end{tabular}

Fonte: MS, 1995

Unidades Federadas, com aquelas apresentadas em anos recentes, por alguns países que apresentam melhor organização da atenção perinatal. Observa-se que o Brasil apresenta a menor taxa de mortalidade neonatal quando comparado ao Chile e ao México, atribuindose esta posição à taxa de mortalidade neonatal tardia em níveis inferiores. Entretanto, a comparação das mesmas taxas com aquelas estimadas para os países desenvolvidos, coloca o Brasil em lugar desfavorável.
Ao comparamos as taxas de mortalidade neonatal precoce de algumas das Unidades Federadas com aquelas de países industrializados, nota-se que os resultados encontrados para estados com melhor oferta de serviços, como por exemplo Rio de Janeiro e São Paulo, apresentam valores bastante elevados. Por outro lado, os achados para os estados do Nordeste são totalmente discrepantes, muito baixos no contexto nacional e quando comparados com as taxas de outros países. 
Tabela 2

Percentual de partos domiciliares, percentual de partos ocorridos na capital, percentual de altas hospitalares de gestantes com menos de vinte e quatro horas e percentual de óbitos registrados nas AlHs das mães.

\begin{tabular}{|c|c|c|c|c|c|}
\hline $\begin{array}{l}\text { Unidade } \\
\text { da Federação }\end{array}$ & $\begin{array}{l}\% \text { de partos } \\
\text { domiciliares }\end{array}$ & $\begin{array}{l}\text { \% de altas } \\
\text { hospitalares } \\
\text { com até } \\
\text { um dia de } \\
\text { permanência }\end{array}$ & $\begin{array}{l}\text { Taxa de } \\
\text { utilização } \\
\text { no período } \\
\text { neonatal } \\
\text { precoce }\end{array}$ & $\begin{array}{l}\text { \% de óbitos } \\
\text { registrados } \\
\text { na AlHs } \\
\text { das mães }\end{array}$ & $\begin{array}{l}\text { Cobertura SUS } \\
\text { em relação aos } \\
\text { nascidos vivos }\end{array}$ \\
\hline Rondônia & 3,6 & 40,5 & 2,9 & 14 & 70 \\
\hline Acre & 12,1 & 50,6 & 0,6 & 26 & 48 \\
\hline Amazonas & 17,4 & 50,9 & 1,3 & 43 & 52 \\
\hline Roraima & 4,7 & 56,3 & 0,5 & 87 & 26 \\
\hline Pará & 14,8 & 33,7 & 1,0 & 29 & 63 \\
\hline Amapá & 7,5 & 51,2 & 0,6 & 0 & 49 \\
\hline Tocantins & 6,7 & 28,6 & 1,5 & 73 & 78 \\
\hline Norte & 11,7 & 39,2 & 1,2 & 35 & 60 \\
\hline Maranhão & 13,9 & 16,0 & 0,5 & 37 & 46 \\
\hline Piauí & 11,2 & 33,4 & 0,4 & 34 & 57 \\
\hline Ceará & 7,1 & 56,4 & 1,1 & 35 & 61 \\
\hline Rio Grande do Norte & 2,8 & 46,7 & 0,6 & 52 & 54 \\
\hline Paraíba & 6,7 & 19,0 & 0,7 & 29 & 52 \\
\hline Pernambuco & 5,7 & 36,3 & 1,0 & 34 & 53 \\
\hline Alagoas & 11,9 & 35,5 & 0,6 & 36 & 45 \\
\hline Sergipe & 7,4 & 42,0 & 0,7 & 61 & 49 \\
\hline Bahia & 14,4 & 46,8 & 1,0 & 55 & 49 \\
\hline Nordeste & 9,9 & 39,6 & 0,8 & 43 & 52 \\
\hline Minas Gerais & 5,5 & 28,5 & 1,5 & 62 & 71 \\
\hline Espírito Santo & 2,5 & 16,1 & 1,4 & 51 & 80 \\
\hline Rio de Janeiro & 1,2 & 10,0 & 1,3 & 59 & 71 \\
\hline São Paulo & 1,1 & 15,2 & 2,3 & 48 & 54 \\
\hline Sudeste & 2,2 & 18,1 & 1,9 & 54 & 63 \\
\hline Paraná & 1,7 & 16,2 & 1,5 & 39 & 63 \\
\hline Santa Catarina & 1,4 & 25,5 & 1,2 & 62 & 66 \\
\hline Rio Grande do Sul & 1,3 & 29,5 & 3,1 & 27 & 62 \\
\hline Sul & 1,5 & 23,1 & 2,0 & 41 & 63 \\
\hline Mato Grosso do Sul & 2,9 & 13,1 & 1,5 & 56 & 90 \\
\hline Mato Grosso & 3,2 & 20,5 & 2,5 & 23 & 85 \\
\hline Goiás & 1,6 & 19,8 & 1,4 & 33 & 83 \\
\hline Distrito Federal & 0,6 & 30,8 & 5,8 & 34 & 85 \\
\hline Centro-oeste & 1,9 & 19,9 & 2,6 & 37 & 83 \\
\hline Brasil & 4,1 & 27,8 & 1,5 & 47 & 51 \\
\hline
\end{tabular}

Fonte: IBGE, 1991, 1998; MS, 1995. 


\begin{tabular}{|c|c|c|c|c|c|c|c|c|c|}
\hline & $\begin{array}{l}\text { Taxa de } \\
\text { natimor- } \\
\text { talidade }\end{array}$ & $\begin{array}{l}\text { Taxa de } \\
\text { mortalid. } \\
\text { neonatal } \\
\text { precoce }\end{array}$ & $\begin{array}{l}\text { Taxa de } \\
\text { mortalid. } \\
\text { neonatal } \\
\text { tardia }\end{array}$ & $\begin{array}{l}\text { Taxa de } \\
\text { mortalid. } \\
\text { neonatal }\end{array}$ & $\begin{array}{l}\text { Cobertura } \\
\text { SUS para } \\
\text { nascidos } \\
\text { vivos }\end{array}$ & $\begin{array}{l}\text { Proporção } \\
\text { de partos } \\
\text { domiciliares }\end{array}$ & $\begin{array}{l}\text { Proporção } \\
\text { de altas } \\
\text { com até } \\
\text { um dia de } \\
\text { internação }\end{array}$ & $\begin{array}{l}\text { Proporção } \\
\text { de óbitos } \\
\text { registrados } \\
\text { nas AlHs } \\
\text { das mães }\end{array}$ & $\begin{array}{l}\text { Taxa de } \\
\text { utilização } \\
\text { no período } \\
\text { neonatal } \\
\text { precoce }\end{array}$ \\
\hline Taxa de natimortalidade & 1,00 & $0,39 *$ & 0,25 & $0,38^{*}$ & $-0,12$ & 0,05 & $-0,29$ & 0,35 & $-0,21$ \\
\hline $\begin{array}{l}\text { Taxa de mortalidade } \\
\text { neonatal precoce }\end{array}$ & $0,39 *$ & 1,00 & $0,72^{\star}$ & $0,99 *$ & 0,23 & $-0,37$ & $-0,26$ & $0,46 *$ & 0,23 \\
\hline $\begin{array}{l}\text { Taxa de mortalidade } \\
\text { neonatal tardia }\end{array}$ & 0,25 & $0,72^{*}$ & 1,00 & 0,81 * & 0,14 & $-0,57^{\star}$ & $-0,29$ & 0,21 & 0,30 \\
\hline $\begin{array}{l}\text { Taxa de mortalidade } \\
\text { neonatal }\end{array}$ & $0,38 *$ & $0,99 *$ & 0,81 * & 1,00 & 0,21 & $-0,43^{*}$ & $-0,27$ & $0,43^{*}$ & 0,26 \\
\hline $\begin{array}{l}\text { Cobertura SUS } \\
\text { de nascidos vivos }\end{array}$ & $-0,12$ & 0,23 & 0,14 & 0,21 & 1,00 & $-0,50^{\star}$ & $-0,57^{*}$ & $-0,07$ & $0,56^{*}$ \\
\hline $\begin{array}{l}\text { Proporção de partos } \\
\text { domiciliares }\end{array}$ & 0,05 & $-0,37$ & $-0,57^{\star}$ & $-0,43^{*}$ & $-0,50$ * & 1,00 & $0,45^{\star}$ & $-0,14$ & $-0,49 *$ \\
\hline $\begin{array}{l}\text { Proporção de altas com } \\
\text { até um dia de internação }\end{array}$ & $-0,29$ & $-0,26$ & $-0,29$ & $-0,27$ & $-0,57^{*}$ & $0,45^{*}$ & 1,00 & $-0,07$ & $-0,22$ \\
\hline $\begin{array}{l}\text { Proporção de óbitos } \\
\text { registrados nas AlHs } \\
\text { das mães }\end{array}$ & 0,35 & $0,46^{*}$ & 0,21 & $0,43^{*}$ & $-0,07$ & $-0,14$ & $-0,07$ & 1,00 & $-0,18$ \\
\hline $\begin{array}{l}\text { Taxa de utilização no } \\
\text { período neonatal precoce }\end{array}$ & $-0,21$ & 0,23 & 0,30 & 0,26 & $0,56^{*}$ & $-0,49$ * & $-0,22$ & $-0,18$ & 1,00 \\
\hline
\end{tabular}

* Correlação significativa no nível de $5 \%$.

\section{Discussão}

Dentre os resultados apresentados neste trabalho, os baixos valores das taxas de natimortalidade e mortalidade neonatal nos estados do Norte e Nordeste precisam ser considerados nesta discussão.

Estes valores em patamar baixo, tanto no contexto nacional como no internacional, podem estar relacionados às proporções de partos domiciliares elevadas nestas Unidades Federadas. Além da correlação inversa expressiva com as coberturas do Sistema Único de Saúde, evidenciada na presente análise, é preciso considerar ainda que os percentuais de partos domiciliares, estimados valendo-se das Estatísticas do Registro Civil, podem estar subestimados devido aos elevados índices de atraso nos registros de nascimentos (IBGE, 1998).

Um outro fator explicativo relaciona-se às diferenças na distribuição das taxas de utilização do SUS no período neonatal precoce, que ressaltam a iniqüidade existente entre as Unidades Federadas, como igualmente concluíram Viacava \& Bahia (1996)ao analisarem os dados da Assistência Médico-Sanitária. As baixas coberturas para os partos e as baixas taxas de utilização no período neonatal do Sistema Único de Saúde evidenciam o apartheid sani- tário ao qual uma fração representativa da população ainda está submetida. Estes fatores, somados à inexpressiva cobertura assistencial (Medicina de Grupo) para as regiões Norte e Nordeste, tal como identificada por Giovanella \& Bahia (1995), parecem ser os mais relacionados aos contrastes Norte/Sul evidenciados.

As proporções elevadas de altas precoces nos procedimentos de parto, ocorridas com até um dia de internação, nas regiões Norte e Nordeste, sugerem possíveis falhas nos processos relacionados à atenção ao parto e puerpério. A fragilidade dos mecanismos de controle e avaliação do SUS podem ser igualmente apontados por estes resultados, uma vez que, segundo a tabela de procedimentos do SIH/SUS, a faixa de variação do tempo de permanência para os procedimentos de parto é de 2 a 6 dias. Desta forma, é surpreendente e inexplicável ter encontrado grandes percentuais para muitas Unidades Federadas, sobretudo se considerarmos que a incompatibilidade do tempo de permanência com o procedimento realizado seria uma das críticas para o controle do faturamento pelo DATASUS, visto que a AIH apresentada nesses casos seria passível de ser rejeitada (glosa da AIH).

O erro de classificação entre nascido vivo e nascido morto, considerado como um dos clás- 
Tabela 4

Componentes da mortalidade neonatal, nos países e Unidades

Federadas estudadas.

\begin{tabular}{|c|c|c|c|c|}
\hline $\begin{array}{l}\text { Países e } \\
\text { Unidades } \\
\text { Federadas }\end{array}$ & Ano & $\begin{array}{l}\text { Taxa } \\
0-6 \text { dias }\end{array}$ & $\begin{array}{l}\text { Taxa } \\
\text { 7-27 dias }\end{array}$ & $\begin{array}{l}\text { Taxa de } \\
\text { mortalidade } \\
\text { neonatal }\end{array}$ \\
\hline Canadá & 1993 & 3,5 & 2,8 & 6,3 \\
\hline Estado Unidos & 1992 & 3,3 & 2,9 & 6,2 \\
\hline Finlândia & 1994 & 2,8 & 1,8 & 4,6 \\
\hline França & 1993 & 2,2 & 4,3 & 6,5 \\
\hline Israel & 1993 & 3,3 & 4,5 & 7,8 \\
\hline Itália & 1992 & 4,6 & 3,3 & 7,9 \\
\hline Portugal & 1994 & 3,4 & 4,6 & 8,0 \\
\hline Inglaterra & 1994 & 3,2 & 3,0 & 6,2 \\
\hline Japão & 1994 & 1,7 & 2,5 & 4,2 \\
\hline México & 1993 & 7,6 & 9,8 & 17,4 \\
\hline Chile & 1992 & 6,0 & 8,3 & 14,3 \\
\hline Brasil & 1995 & 8,3 & 2,2 & 10,6 \\
\hline Rio de Janeiro & 1995 & 9,1 & 2,2 & 11,3 \\
\hline São Paulo & 1995 & 12,0 & 3,0 & 15,0 \\
\hline Minas Gerais & 1995 & 10,4 & 2,2 & 12,6 \\
\hline Rio Grande do Sul & 1995 & 8,0 & 3,2 & 11,2 \\
\hline Maranhão & 1995 & 3,4 & 0,9 & 4,4 \\
\hline Piauí & 1995 & 2,6 & 0,8 & 3,4 \\
\hline Ceará & 1995 & 8,8 & 2,2 & 11,1 \\
\hline Distrito Federal & 1995 & 7,5 & 2,5 & 10,0 \\
\hline Pernambuco & 1995 & 7,6 & 2,5 & 10,1 \\
\hline
\end{tabular}

Fonte: MS, 1995; WHO, 1995 os óbitos registrados nas AIHs das mães provavelmente representam a demanda reprimida das internações de recém-nascidos prematuros e extremamente imaturos em leitos de UTI neonatal e Unidades intermediárias.

Dentre as limitações para o estudo mais detalhado das mortes infantis no presente trabalho está a ausência das variáveis "peso" e "idade gestacional” nos formulários AIH. Apesar de esta situação ter sido contornada a partir de junho de 1998, com a inclusão destas variáveis nos campos a serem preenchidos nos procedimentos de parto (Brasil, 1998), para a presente análise, estas variáveis ainda não estavam disponíveis.

Selecionando-se as Unidades Federadas das regiões Sul e Sudeste, que possuem uma melhor cobertura de serviços obstétricos e neonatais, para comparação com países desenvolvidos, pôde-se verificar que as taxas de mortalidade neonatal e de seus componentes espelham os graves problemas da atenção ao nascimento, com valores em patamar muito alto.

Conforme Butterfield (1993) e Kollee et al. (1992), dos problemas a serem enfrentados para reorganizar a atenção obstétrica e neonatal assumem relevância: a segurança das gestantes e recém-nascidos e o acesso/cobertura dos serviços. Os principais aspectos relacionados ao primeiro, referem-se essencialmente à identificação dos diferentes riscos da gravidez pela assistência pré-natal e a transferência "antenatal” das gestantes de risco para serviços hospitalares de maior complexidade. Já em relação ao segundo, a principal estratégia centra-se na oferta obstétrico-pediátrica disponíveis às parturientes próximas ao local de residência.

Os achados desta análise apontam para grandes lacunas em ambos. Os limites impostos pela baixa oferta e pelo acesso aos serviços do SUS são restrições relevantes à população residente nas regiões Norte e Nordeste. Por outro lado, a qualidade da assistência se reflete nas elevadas taxas de mortalidade infantil precoce nas regiões mais desenvolvidas do país.

É importante enfatizar que o aprimoramento da qualidade das informações do SIH/SUS é um tema que tem merecido pouca atenção por parte dos técnicos de saúde, por tratar-se de um banco de dados que tem sido usado quase que exclusivamente para o faturamento, com poucas exceções na área de investigação (Veras, 1992; Gouvêa, 1996). A utilização do SIH/SUS como banco dados de investigação na pesquisa epidemiológica, certamente identificará os problemas relacionados à fidedignidade dos registros e permitirá desenvolver as ações necessárias para qualificar as informações. 
A utilização do SIH/SUS, como base de dados de referência para construção de indicadores a serem utilizados no acompanhamento da atenção básica, representa a possibilidade de identificar questões a serem enfrentadas para reestruturar a atenção obstétrica e neonatal. Em particular, no caso das Unidades Federadas que possuem uma boa cobertura de serviços obstétricos e neonatais, o SIH/SUS que é disponibilizado rapidamente pelo DATASUS sem

\section{Referências}

ARAGÃO, M. G., 1994. Mortalidade Perinatal na Região Metropolitana do Rio de Janeiro, 1979-1989. Dissertação de Mestrado, Rio de Janeiro: Escola Nacional de Saúde Pública, Fundação Oswaldo Cruz.

BARROS, F.; VICTORIA, C. G.; TEIXEIRA, A. M. B. \& PUERTO FILHO, M., 1985. Mortalidade perinatal e infantil em Pelotas, Rio Grande do Sul: Nossas estatísticas são confiáveis? Cadernos de Saúde Pública, 1:348-358.

BARROS, F; VICTORIA, C. G. \& VAUGHAN, J. P., 1987. Causas de mortalidade perinatal em Pelotas, RS (Brasil). Utilização de uma classificação simplificada. Revista de Saúde Pública, 21:310-316.

BECKER, R. A., 1991. Análise da Mortalidade-Delineamentos Básicos. Brasília: Coordenação de Informações Epidemiológicas, Fundação Nacional de Saúde, Ministério da Saúde.

BERCINI, L. O., 1994. Mortalidade neonatal de residentes em localidade urbana da Região sul do Brasil. Revista de Saúde Pública, 28:38-45.

BRASIL, 1998. Portaria no 2.817, de 29 de maio de 1998. Brasília: Diário Oficial da República Federativa do Brasil, n. 103, p. 48, 2 jun. Seção 1.

BUTTERFIELD, L. J., 1993. Historical perspectives of neonatal transport. Pediatric Clinics of North America, 40:221-239.

CAMPBELL, R. \& MacFARLANE, A., 1986. A place of delivery: A review. British Journal of Obstetrics and Ginaecology, 93:675-683.

CARVALHO, M. L., 1993. Mortalidade Neonatal e Aspectos da Qualidade da Atenção a Saúde na Região Metropolitana do Rio de Janeiro em 19861987. Dissertação de Mestrado, Rio de Janeiro: Escola Nacional de Saúde Pública, Fundação Oswaldo Cruz. a defasagem de quase dois anos do Sistema de Mortalidade (SIM/MS), permite o acompanhamento das mortes neonatais e da natimortalidade em um espaço curto de tempo.

Assim, o aprofundamento das análises do SIH/SUS poderá, com certeza, contribuir para acompanhamento espaço-temporal destas taxas, fornecendo subsídios para ações de organização do programa materno-infantil.
GIOVANELLA, L. \& BAHIA, L., 1995. Saúde: O poder da atenção pública. Saúde em Debate, 46:8-13.

GOUVÊA, C. S. D., 1996. Tendências Recentes da Assistência Hospitalar no Estado do Rio de Janeiro (1992-1995). Dissertação de Mestrado, Rio de Janeiro: Escola Nacional de Saúde Pública, Fundação Oswaldo Cruz.

GROUPE IMAGE, 1997. Groupe d'Animation et Impulsion National. L'Obstétrique en France. Paris: École National de Santé Públique.

IBGE (Fundação Instituto Brasileiro de Geografia e Estatística), 1991. Censo Demográfico. Rio de Janeiro: IBGE.

IBGE (Fundação Instituto Brasileiro de Geografia e Estatística), 1998. Estatísticas do Registro Civil para o Ano 1995. Rio de Janeiro: IBGE.

KOLLEE, L. A. A.; BRAND, R.; SCHREUDER, A. M.; ENS-DOKKUM, M. H.; VEEN, S. \& VERLOOVEVANHORICK, S. P., 1992. Five-year outcome of preterm and very low birth weight infants: A comparison between maternal and neonatal transport. Obstetrics \& Gynecology, 80:635-638.

LEAL, M. C. \& SZWARCWALD, C. L., 1996. Evolução da mortalidade neonatal no Estado do Rio de Janeiro, Brasil (1979 a 1993): Análise por causa segundo grupo de idade e região de residência. $\mathrm{Ca}$ dernos de Saúde Pública, 12:243-252.

MELO, E. C. P., 1996. Distribuição Espacial da Doença Isquêmica do Coração na Região Sudeste-Brasil: Mortalidade, Internação Hospitalar, Oferta de Serviços de Saúde e Perfil Sócio-econômico. Dissertação de Mestrado, Rio de Janeiro: Escola Nacional de Saúde Pública, Fundação Oswaldo Cruz.

MS (Ministério da Saúde), 1995. AIH - Sistema de Apoio à Entrada de Dados. Manual das Unidades Hospitalares. Brasília: Secretaria Nacional de As- 
sistência à Saúde, Instituto Nacional de Assistência Médica da Previdência Social, Sistema Único de Saúde.

OMS (Organização Mundial de Saúde), 1985. Classificação Internacional de Doenças - 9a Revisão. v. 1. São Paulo: OMS.

RUTSTEIN, D. D.; MERENBERG, W.; CHALMERS, T. C.; CHILD, C. G.; FISHMAN, A. P. \& PERRIN, E. B., 1976. Measuring the quality of medical care: A clinical method. New England Journal of Medicine, 294:582-588.

SALINAS, A. M.; CORIA, I.; REYES, H. \& ZAMBRANA, M., 1997. Effect of quality of care on preventable perinatal mortality. International Journal for Quality in Health Care, 9:93-99.

SAS INSTITUTE, 1998. The SAS System, Version 6.12. Raleigh: SAS Institute.

SCHRAMM, J. M. A. \& SZWARCWALD, C. L., 2000. O Sistema de Informações Hospitalares como fonte de informações para estimar a mortalidade neonatal e a natimortalidade. Revista de Saúde Pública, 34:272-279.

SHENAI, J. P.; MAJOR, C. W.; GAYLORD, M. S.; BLADE, W. W.; SIMMONS, A.; OLIVER, S. \& DE ARMOND, D. A., 1991. A successful decade of regionalized perinatal care in Tennessee: The neonatal experience. Journal of Perinatology, 40: 137-143.
SOUZA, R. T. S. \& GOTLIEB, S. L. D., 1993. Probabilidade de morrer no primeiro ano de vida em área urbana da Região Sul, Brasil. Revista de Saúde Pública, 27:445-543.

SPSS INCORPORATION, 1995. SPSS for Windows: A Base System User's Guide. Release 8.0. Chicago: SPSS Incorporation.

SZWARCWALD, C. L., 1997. Apreciação das Informações de Registro sobre Nascimentos e Óbitos no Brasil. Recife: Seminário sobre a Mortalidade Infantil no Nordeste do Brasil. (mimeo.)

UN (United Nations), 1983. Manual X: Indirect Techniques for Demographic Estimation. New York: UN.

VERAS, C. T., 1992. Equity in the Use of Private Hospitals Contracted by Compulsory Insurance Scheme in the City of Rio de Janeiro, Brazil, in 1986. Ph. D. Thesis, London: London School of Economics and Political Science, London University.

VIACAVA, F. \& BAHIA, L., 1996. Assistência médicosanitária. Os serviços de saúde segundo o IBGE. Dados/Fiocruz, 20.

WHO (World Health Organization), 1995. World Health Statistics, 1990-1994. Genève: WHO. 